\title{
La théorie de l'attachement et les pratiques périnatales
}

\author{
R. Dugravier $\cdot$ J. Wendland
}

(C) Lavoisier SAS 2020

Longtemps, la théorie de l'attachement est restée confidentielle en France. John Bowlby, pédopsychiatre anglais à l'origine de cette théorie, avait souhaité dès le début développer une approche construite sur les connaissances les plus récentes (les compétences précoces du nourrisson, la théorie des systèmes motivationnels, les apports de l'éthologie) et sur une solide méthode expérimentale. Ses premiers travaux avaient pour objet de démontrer les conséquences des séparations parent-enfant, en particulier quand ceux-ci sont très jeunes. Alors qu'avant même les années 1950 il travaillait déjà en recherche et en clinique avec une équipe française, ses concepts sont restés ignorés (Colloque imaginaire, 1974, René Zazzo) ou englués dans une controverse stérile.

Ce n'est qu'au début des années 2000, non sans peine, que cette théorie a pris une place plus importante au sein de la communauté pédopsychiatrique grâce aux efforts et à l'engagement de Nicole et Antoine Guedeney. Cette théorie est depuis devenue incontournable dans d'autres domaines tels que la protection de l'enfance, les modes d'accueil du jeune enfant et dans le champ de la périnatalité, donnant un nouveau souffle au travail clinique et à la recherche.

C'est donc une théorie construite à partir de la nécessité pour l'enfant, dès la naissance, de nouer une relation d'attachement avec un adulte qui répond à ses besoins de proximité et de protection. Le système d'attachement de l'enfant est ainsi activé par toute situation de stress, peur ou inconfort, et celui-ci recourt alors à tout un panel de comportements pour obtenir tant que possible une proximité avec sa figure d'attachement (tout adulte engagé dans une relation continue, durable, affective et sensible avec l'enfant) afin

\section{R. Dugravier $(\square)$}

Centre de psychopathologie périnatale du boulevard Brune,

Pôle 14, GHU Paris psychiatrie et neurosciences,

26, boulevard Brune, F-75014 Paris, France

e-mail : r.dugravier@ghu-paris.fr

\section{J. Wendland $(\bowtie)$}

Laboratoire de psychopathologie et processus de santé, université de Paris, 71, avenue Édouard-Vaillant,

F-92774 Boulogne-Billancourt, France

e-mail : jaqueline.wendland@parisdescartes.fr

Unité petite enfance et parentalité Vivaldi,

F-75012 Paris, France d'obtenir du réconfort. Les réponses du parent, sa manière de prodiguer les soins, c'est-à-dire son système de caregiving, vont modeler le style d'attachement de l'enfant, objectivable dès l'âge d'un an. On parle ainsi, en s'appuyant sur le modèle expérimental de la situation étrange (séquences de séparations et de retrouvailles entre l'enfant et sa figure d'attachement), d'attachements sécure ou insécure, considérés comme des stratégies adaptatives plus ou moins souples. Ce n'est qu'en grandissant que l'enfant acquiert des représentations d'attachement tirées de ces expériences pour établir un modèle de soi (digne ou non de recevoir des soins, confiant ou méfiant), d'autrui (fiable, disponible ou non) et du monde.

Rappelons que le système d'attachement ne se comprend que dans un équilibre avec le système exploratoire, moteur de la curiosité, de l'exploration et de la découverte du monde.

Ainsi, la mère, le père (les figures d'attachement) jouent tour à tour le rôle d'une base de sécurité qui soutient l'exploration et d'un havre de sécurité qui accueille l'enfant quand il a besoin d'être réconforté.

Dans ce numéro, nous avons choisi de présenter divers dispositifs, réflexions ou expériences en périnatalité qui prennent appui sur la théorie de l'attachement.

Le premier article (Romain Dugravier et Marie-Noëlle Vacheron), illustration d'une démarche préventive et novatrice, présente la consultation CICO destinée aux femmes avec un trouble psychique ayant un désir d'enfant ou étant enceintes. Cette consultation d'experts apporte conseil sur les meilleurs choix thérapeutiques et aide à la construction du parcours de grossesse et des premiers temps après la naissance.

Ensuite, Lise Michel et Jaqueline Wendland abordent les enjeux de l'activation des systèmes d'attachement et de caregiving chez les femmes au cours de la période périnatale, apportent des précisions sur les concepts d'attachement prénatal et de bonding et soulignent le rôle de la relation aux soignants durant cette période de vulnérabilité.

Jean Sarlangue présente ensuite le cordon numérique, une expérience originale d'envoi de courtes vidéos aux parents d'enfants hospitalisés en néonatalogie dans le but d'aider à l'établissement et au maintien du lien des parents avec leur 
nouveau-né. Le choix de la vidéo est alors un geste thérapeutique en lui-même.

À son tour, Frédéric Van der Borght expose le dispositif d'accueil familial comme soutien des premiers temps de la parentalité et de l'attachement destiné à accompagner des parents vulnérables au sein des centres Aire de famille.

Les principes et apports de la visite à domicile, en PMI ainsi qu'en recherche, comme outil de prévention, de soin et de soutien à l'attachement sont ensuite présentés conjointement par une puéricultrice, Augusta Bonnard, et une pédopsychiatre, Lola Villetorte.
Enfin, Bérengère Beauquier-Maccotta, Christine Desvignes et Sandra Rusconi Serpa explicitent les principes de la guidance interactive, un dispositif thérapeutique fondé sur de courtes séquences d'interactions parent-bébé filmées. Ces séquences sont ensuite reprises avec les parents afin de les amener à mieux lire les signaux et besoins de leur enfant et de progressivement modifier les patterns interactifs dysfonctionnels qui peuvent avoir des répercussions pour les relations d'attachement. Peu développée en France, cette pratique offre des perspectives prometteuses de soin. 